\title{
Association of existing diabetes, gestational diabetes and glycosuria in pregnancy with macrosomia and offspring body mass index, waist and fat mass in later childhood: findings from a prospective pregnancy cohort
}

\author{
D. A. Lawlor • A. Fraser • R. S. Lindsay • A. Ness • \\ D. Dabelea • P. Catalano • G. Davey Smith • N. Sattar • \\ S. M. Nelson
}

Received: 28 April 2009 /Accepted: 3 September 2009/Published online: 20 October 2009

(C) Springer-Verlag 2009

\author{
Abstract \\ Aims/hypothesis The aim of the study was to examine the \\ association of existing diabetes (i.e. already diagnosed prior \\ to pregnancy), gestational diabetes and glycosuria (both \\ diagnosed and ascertained during pregnancy) with birth- \\ weight and future offspring BMI, waist circumference and \\ fat mass (assessed by dual x-ray emission absorptiometry). \\ D. A. Lawlor $(\varangle) \cdot$ A. Fraser $\cdot$ G. Davey Smith \\ MRC Centre for Causal Analyses in Translational Epidemiology, \\ Department of Social Medicine, University of Bristol, \\ Oakfield House, Oakfield Grove, \\ Bristol BS8 2BN, UK \\ e-mail: d.a.lawlor@bristol.ac.uk \\ R. S. Lindsay $\cdot$ N. Sattar \\ BHF Glasgow Cardiovascular Research Centre, \\ Faculty of Medicine, University of Glasgow, \\ Glasgow, UK \\ A. Ness \\ Department of Oral and Dental Science, University of Bristol, \\ Bristol, UK \\ D. Dabelea \\ Department of Epidemiology, Colorado School of Public Health, \\ University of Colorado, \\ Denver, CO, USA \\ P. Catalano \\ Department of Reproductive Biology, \\ Case Western Reserve University, \\ Cleveland, $\mathrm{OH}$, USA \\ S. M. Nelson \\ Department of Reproductive and Maternal Medicine, \\ Faculty of Medicine, University of Glasgow, \\ Glasgow, UK
}

Methods A prospective pregnancy/birth cohort study was performed using data from the Avon Longitudinal Study of Parents and Children.

Results Among 10,591 mother-offspring pairs included in analyses with birth size, women with existing diabetes $(n=40)$, those diagnosed with gestational diabetes $(n=53)$ and those with at least two episodes of ++ glycosuria $(n=372)$ had greater mean birthweight and odds for macrosomia (birthweight $>4,000 \mathrm{~g}$ ) than women with none of these. Adjusted odds ratios for macrosomia were 3.56 (95\% CI 1.53-8.28), 5.50 (95\% CI 1.18-10.30) and 1.58 (95\% CI 1.18-2.12) for existing diabetes, gestational diabetes and glycosuria, respectively. Among 6,842 motheroffspring pairs with anthropometric measurements at age 9 11 years, maternal gestational diabetes and glycosuria (but not existing diabetes) were associated with increased offspring odds of general or central overweight/obesity. For gestational diabetes, these associations attenuated towards the null with adjustment for maternal prepregnancy BMI, but independent associations remained for glycosuria. The adjusted odds ratio for general overweight/obesity when comparing women with at least two episodes of ++ glycosuria with those with no evidence of diabetes or glycosuria was $1.35(95 \%$ CI $1.00-1.82)$ and that for central obesity (top $10 \%$ waist circumference vs all others) was 1.31 (95\% CI 1.00-1.72).

Conclusions/interpretation These results provide some evidence for a long-term effect of maternal glycaemia in pregnancy on offspring obesity risk.

Keywords ALSPAC - Epidemiology · Fetal overnutrition · Gestational diabetes $\cdot$ Glycosuria $\cdot$ Obesity 


\section{Abbreviations \\ ALSPAC Avon Longitudinal Study of Parents and Children \\ DXA Dual x-ray emission absorptiometry \\ LGA Large for gestational age}

\section{Introduction}

Gestational diabetes is associated with higher birthweight and greater fetal adiposity [1, 2]. Among non-diabetic mothers there is a linear association between fasting and postchallenge glucose and greater birth size [3]. Fuelmediated teratogenesis (also known as fetal or developmental overnutrition) has been suggested as the most likely mechanism for these associations $[4,5]$.

The resulting fetal overnutrition may also programme offspring to life-long increased adiposity [6]. In studies of Pima Indians, a marked excess in the risk of obesity has been found at all ages from birth to 20 years in offspring born to mothers who had diabetes during their pregnancy compared with the offspring of either mothers who developed diabetes later in their lives or those who never developed diabetes [7-9]. In a nuclear family study, also conducted in Pima Indians, obesity was greater among offspring born after the mother had been diagnosed with diabetes than in their sibs born before their mother's diagnosis [10]. These differences were not found in siblings born before and after the father was diagnosed with type 2 diabetes, suggesting that, in a population at high risk of obesity and diabetes, exposure to maternal diabetes in utero has long-term effects on the offspring that are in addition to genetic and shared familial factors. If intrauterine exposure to gestational diabetes is causally related to later offspring obesity via intrauterine pathways, this could result in an acceleration of diabetes and obesity risk that would continue across several generations even with improvements to the obesogenic environment [11]. Given the limited evidence from non-Pima populations for associations of gestational diabetes/hyperglycaemia with the longterm risk of offspring obesity, further study in these populations is required [6].

In countries where universal screening for gestational diabetes is not undertaken by fasting or random glucose or glucose challenge testing, the presence of glycosuria has been used as screening method [12]. This approach is relatively cheap, non-invasive and acceptable to pregnant women [12]. However, most national guidelines [13, 14] do not recommend it as a screening tool. These guidelines give its low sensitivity for identifying hyperglycaemia/gestational diabetes determined by the glucose tolerance test as a reason for not supporting its use [15]. However, the is a paucity of evidence on the sensitivity and specificity of glycosuria for gestational diabetes screening. One retrospective study of 3,217 women demonstrated a sensitivity of $7 \%$, specificity of $99 \%$ and positive and negative predictive values of $13 \%$ and $97 \%$ respectively [16]. These findings are similar to those of a more recent prospective study of 1,001 women [17]. In a study examining prescreening based on risk factors, the strongest predictor of gestational diabetes was glycosuria, with an odds ratio of 9 , with capillary fasting glucose having a null odds ratio and high BMI, family history, previous gestational diabetes and macrosomia having odds ratios between 1.5 and 4 [18]. To our knowledge, few previous studies have examined the association of glycosuria with macrosomia and none have examined its association with future offspring obesity. This is important since adverse perinatal and long-term outcomes are at least as important as a diagnosis of gestational diabetes.

The aim of this study was to examine the association of existing diabetes, gestational diabetes and glycosuria in pregnancy with birthweight, macrosomia and future offspring BMI, waist circumference and fat mass, assessed by dual $\mathrm{x}$-ray emission absorptiometry (DXA) at ages 9-11 years in a large UK general populationbased study.

\section{Methods}

Study population and general procedure The Avon Longitudinal Study of Parents and Children (ALSPAC) is a longitudinal, population-based birth cohort study that recruited 14,541 pregnant women resident in Avon, in the south west of England with expected date of delivery between 1 April 1991 and 31 December 1992 (www.alspac. bris.ac.uk) [19]. ALSPAC aims to examine the genetic and environmental (including intrauterine) determinants of development and health. Participants are representative of pregnant women in the Avon area in the 1990s and similar to women of reproductive age across the UK [19]. We restricted our analyses to mother-offspring pairs that were singleton pregnancies and live births who survived to at least 1 year of age $(n=13,678)$. Of the 13,678 live-born singleton infants who survived to 1 year, $10,725(79 \%)$ had complete data on maternal diabetes/glycosuria status during pregnancy. These 10,725 mother-offspring pairs formed our eligible cohort for analyses. Of these 10,725 pairs, $10,591(99 \%)$ had complete data on birthweight and were used in the analyses examining associations with birthweight and macrosomia.

Ethics approval for all aspects of data collection was obtained from the ALSPAC Law and Ethics Committee (IRB 00003312) and the local research ethics committee. 
From age 7, surviving offspring, with parental consent, were invited to regular follow-up clinics at which height, weight and waist circumference were assessed; from age 9, fat mass was assessed by DXA. Among the 10,725 eligible mother-offspring pairs, $6,771(63 \%)$ of the offspring had data on fat mass, 6,842 (64\%) had data on BMI and 6,854 $(64 \%)$ had data on waist circumference assessed between ages 9 and 11 years. These groups formed the analysis participants for examining associations with later childhood outcomes. Compared with the original recruited population, children attending the clinic were more likely to be from a higher socioeconomic group and have more educated parents and older mothers [19].

At recruitment, women were asked about existing diabetes and any previous history of gestational diabetes. Research midwives using a standard protocol abstracted information on clinical diagnoses of gestational diabetes and glycosuria for the index pregnancy from the antenatal, pregnancy and postnatal medical records. The midwives searched all aspects of these records and gestational diabetes was defined as any record of a diagnosis of gestational diabetes at any time during the pregnancy in women without existing diabetes at the start of pregnancy. At the time that this study was undertaken, UK policy was for women who had glycosuria and/or risk factors (obesity, family history of diabetes, previous gestational diabetes or macrosomia, and women from ethnic groups at high risk) to be offered further testing, which could include a fasting glucose or oral glucose tolerance test. Information on glycosuria (recorded as none, trace,,,++++++ or more) was abstracted from the records of each antenatal clinic visit made by the woman (median number, 14 per woman). UK practice at the time of this study was for all pregnant women to have urine tested for glycosuria and proteinuria at every antenatal clinic visit. Glycosuria was defined as a record of at least ++ (equal to $13.9 \mathrm{mmol} / 1$ or $250 \mathrm{mg} / 100 \mathrm{ml}$ ) on at least two occasions at any time during the pregnancy. Because some level of glycosuria is common in pregnancy as a result of increased glomerular filtration rate [20], $250 \mathrm{mg} / 100 \mathrm{ml}$ is the threshold that has generally been used for indicating the need for further testing to diagnose gestational diabetes $[16,17]$. Using these data, we classified the women into one of four mutually exclusive categories: no evidence of glycosuria or diabetes (hereafter referred to as 'healthy women'); existing diabetes before the pregnancy; gestational diabetes (i.e. a diagnosis in the medical records of gestational diabetes in any woman with no history of existing diabetes); and glycosuria (i.e. ++ glycosuria on two occasions in women with no evidence of existing or gestational diabetes).

Gestational age and infant birthweight were recorded in the delivery room and abstracted from obstetric records and/or birth notifications. Birthweight standard deviation (z-) scores, standardised internally for sex and gestational age (in completed weeks) were calculated. Macrosomia was defined as a birthweight $>4,000 \mathrm{~g}$ and large for gestational age (LGA) was defined as $>90$ th centile of the birthweight for sex and gestational age $z$ score.

Identical protocols were used at both the 9- and 11-year follow-up clinics. Weight and height were measured in light clothing and without shoes. Weight was measured to the nearest $0.1 \mathrm{~kg}$ using Tanita scales (Porter Nash Medical, London, UK). Height was measured to the nearest $0.1 \mathrm{~cm}$ using a Harpenden stadiometer. Waist was measured to the nearest $1 \mathrm{~mm}$ at the mid-point between the lower ribs and the pelvic bone with a flexible tape. A narrow fan beam densitometer (Lunar Prodigy; GE Healthcare Luncar Ltd, Cambridge, UK) was used to perform a whole-body DXA scan, in which bone content, lean and fat mass were measured. For all analyses with offspring BMI, waist circumference and fat mass as the outcome, we used sex and age (in month categories) standard deviation scores ( $z$ scores). As in a previous study using these data [21], we completed analyses with a combined outcome of 9- or 11 -year $z$ score (i.e. we included participants if they had attended and completed assessments at either the 9- or 11-year follow-up assessment, using the 9-year assessment for all who had these measurements and the 11-year assessment for those who did not have a 9-year measurement). Among those participants with assessments at both clinics, measurements at each clinic were highly correlated (Pearson's correlation coefficient was 0.93 for BMI, 0.88 for waist and 0.93 for fat mass). General overweight/obesity was defined using the International Obesity Taskforce age- and sex-specific thresholds for childhood BMI [22], and central obesity was defined as an age- and sex-specific waist circumference $\geq 90$ th percentile [23], based on waist circumference percentile curves derived for British children [24].

Maternal parity, mode of delivery and the child's sex were obtained from the obstetric records. Based on questionnaire responses, the highest parental occupation was used to allocate the children to family social class groups (classes I [professional/managerial] to V [unskilled manual workers], using the 1991 British Office of Population and Census Statistics classification). At enrolment, the mother was asked to record her height and prepregnancy weight, from which prepregnancy BMI was calculated. Mothers were asked about their smoking throughout pregnancy and these data were used to generate a categorical variable: never smoked prior to or during pregnancy; smoked only in prepregnancy or in the first trimester; smoked throughout pregnancy (reported smoking in second and/or third trimester).

Statistical analyses We used multivariable linear regression to examine the association of maternal glycosuria/diabetes 
with offspring anthropometry, controlling for potential confounders. We adjusted for the number of urine tests that the woman had completed during her pregnancy. Number of urine tests is unlikely to be related to outcomes (and therefore unlikely to confound associations), but may contribute to exposure measurement error, since women who have more clinic visits and hence more urine tests will have a greater opportunity for fulfilling our criteria or two or more episodes of ++ elevation of glycosuria. In models with fat mass as the outcome, we adjusted for height and height squared (assessed at the same time as the DXA scan) so that we had a measure of association with fat mass that was not determined by height [21].

Within each of our main analysis datasets $(n=10,591$ for birthweight/macrosomia/LGA and $n=6,771-6,854$ for fat mass) there were small amounts of missing data on individual confounding factors. For most confounding variables, between $0 \%$ and $5 \%$ had missing data; head of household social class had most missing data $(9.5 \%)$. For the main multivariable analyses, in which we adjusted for potential confounding factors, $8,519(80 \%)$ of the 10,591 pairs included in the birthweight/macrosomia analyses and $5,807-5,893(86 \%)$ of the $6,771-6,854$ included in the later outcome analyses had complete data on all potential confounding factors. We used multiple multivariate imputation [25], using all other covariables, to impute values for missing covariable data in both of our main analyses. We repeated all analyses including only those with complete data (without imputation) and found no substantive difference between those analyses and the ones presented here with multiple imputations.

All analyses were conducted using Stata version 10.1 (Stata Inc., College Station, TX, USA). Values are mean \pm SD unless stated otherwise.

\section{Results}

Of the 10,591 mother-offspring pairs included in the birthweight analyses, 10,126 (96\%) were defined as healthy; $40(0.4 \%)$ had existing diabetes; $53(0.5 \%)$ were diagnosed with gestational diabetes (of whom 19 had experienced gestational diabetes in one previous pregnancy) and $372(3.5 \%)$ had glycosuria. Similar proportions of women were in each category among the 6,771 motheroffspring pairs included in the fat mass analyses: 6,478 $(96 \%)$ had no evidence of diabetes or glycosuria; $26(0.4 \%)$ had existing diabetes; $38(0.6 \%)$ had gestational diabetes and 229 (3.4\%) had glycosuria. Proportions of mothers with diabetes or glycosuria were also the same for offspring with data on BMI and waist circumference at age 911 years. Women diagnosed with gestational diabetes had more episodes of ++ glycosuria than healthy women (mean difference in number of episodes of ++ glycosuria, 1.70, 95\% CI 1.47-1.93). Of the total of 40 mothers with existing diabetes $31(77.5 \%)$ reported that prior to their pregnancy they were being treated with insulin, two were being treated with oral hypoglycaemic agents and six with diet only (one did not provide treatment information). The median age at diagnosis of diabetes (data available for all 40) in these women was 12 years (interquartile range 9-22) and all were diagnosed before age 29 .

Mean birthweight was $3,363 \pm 510 \mathrm{~g}$ in females and $3,476 \pm 559 \mathrm{~g}$ in males, with $552(10 \%)$ of females and 874 $(16 \%)$ of males being macrosomic. Mean BMI and waist circumference at age 9 years in females and males respectively were $17.9 \pm 3.0 \mathrm{~kg} / \mathrm{m}^{2}$ and $17.5 \pm 2.8 \mathrm{~kg} / \mathrm{m}^{2}$, and $626 \pm 79 \mathrm{~mm}$ and $632 \pm 77 \mathrm{~mm}$. Twenty three percent $(1,570)$ of the participants were overweight or obese at age 9-11 years; overweight or obesity was more common in females than males $(25 \%$ vs $21 \%, p<0.0001)$. Thirty-eight per cent $(2,614)$ of the participants were centrally obese at age 9-11 years; central obesity was more common in females compared with males $(42 \%$ vs $35 \%, p<0.0001)$. Median fat mass at age 9-11 years was 8,494 g (interquartile range 5,953-12,160 g) in females and 5,790 g $(3,997-9,248 \mathrm{~g})$ in males. By age 11 years, the mothers of this cohort reported that 13 (six male) offspring were on treatment for diabetes; all were using insulin.

In univariable analyses, maternal BMI was weakly positively correlated with birthweight $(r=0.17, p<0.00001)$ and modestly positively correlated with offspring BMI, waist and fat mass (all $r \sim 0.3 ; p<0.00001$ for all). As reported previously in this cohort [26], birthweight was weakly positively correlated with offspring BMI, waist and fat mass (all $r \sim 0.1 ; p<0.00001$ for all). Offspring BMI, waist and fat mass at age 9-11 were all strongly positively correlated with each other (all $r>0.9 ; p<0.00001$ for all).

Table 1 shows participant characteristics by maternal diabetes/glycosuria status.

Table 2 presents the multivariable associations of maternal diabetes/glycosuria status with offspring birthweight and macrosomia. Results for LGA were essentially the same as those presented for macrosomia and are therefore not presented. Existing diabetes, gestational diabetes and glycosuria were all associated with greater mean birthweight and with odds of macrosomia. Adjustment for potential confounding by maternal age, social class, parity, smoking in pregnancy and mode of delivery resulted in modest attenuation (model 2). Inclusion of maternal prepregnancy BMI resulted in more marked attenuation (model 3); however, the observed positive associations with birthweight and macrosomia remained (Table 2). Additional adjustment for number of urine tests in pregnancy did not alter any of the results presented in Table 2. 
Table 1 Participant characteristics by maternal diabetes/glycosuria status in pregnancy

\begin{tabular}{|c|c|c|c|c|c|c|}
\hline \multirow[t]{2}{*}{ Characteristic } & \multirow{2}{*}{$\begin{array}{l}\text { Number } \\
\text { with data }\end{array}$} & \multicolumn{4}{|c|}{ Mean (SD) or number (\%) by diabetes/glycosuria status } & \multirow[t]{2}{*}{$p$ value $^{\mathrm{a}}$} \\
\hline & & $\begin{array}{l}\text { Healthy } \\
(n=10,126)\end{array}$ & $\begin{array}{l}\text { Existing } \\
\text { diabetes } \\
(n=40)\end{array}$ & $\begin{array}{l}\text { Gestational } \\
\text { diabetes } \\
(n=53)\end{array}$ & $\begin{array}{l}\text { Glycosuria } \\
(n=372)\end{array}$ & \\
\hline \multicolumn{7}{|l|}{ Maternal characteristics at time of pregnancy } \\
\hline Manual social class & 9,580 & $1,645(18)$ & $6(15)$ & $10(22)$ & $78(24)$ & 0.048 \\
\hline One or more previous pregnancies $(n)$ & 10,334 & $545(6)$ & 0 & $8(16)$ & $29(8)$ & 0.005 \\
\hline Mother smoked throughout pregnancy $(n)$ & 10,466 & $2,384(24)$ & $7(18)$ & $4(8)$ & $90(24)$ & 0.016 \\
\hline Mother's age at birth (mean, years) & 10,591 & $28.2(4.9)$ & $28.8(3.8)$ & $29.7(5.0)$ & $28.2(4.9)$ & 0.166 \\
\hline Mother's BMI (mean, $\mathrm{kg} / \mathrm{m}^{2}$ ) & 9,988 & $22.9(3.8)$ & $24.2(4.2)$ & $26.6(6.4)$ & $23.8(4.4)$ & $<0.0001$ \\
\hline Maternal obesity $(n)$ & 9,988 & $520(5)$ & $2(6)$ & $9(19)$ & $31(9)$ & 0.0007 \\
\hline Caesarean section $(n)$ & 10,572 & $1,151(11)$ & $21(53)$ & $17(33)$ & $49(13)$ & $<0.0001$ \\
\hline $\begin{array}{l}\text { Number of urine tests (mean, interquartile } \\
\text { range) }\end{array}$ & 10,591 & $12(10-14)$ & $14(11-17)$ & $14(12-14)$ & $14(12-17)$ & $<0.0001$ \\
\hline \multicolumn{7}{|l|}{ Offspring characteristics at birth } \\
\hline Female $(n)$ & 10,591 & 4,998 (49) & $19(48)$ & $20(38)$ & $202(53)$ & 0.146 \\
\hline Birth weight (mean, g) & 10,591 & $3,416(536)$ & $3,248(787)$ & $3,711(655)$ & $3,511(534)$ & $<0.0001$ \\
\hline Gestational age (mean, weeks) & 10,591 & $39.5(1.9)$ & $37.5(2.6)$ & $38.2(1.9)$ & $39.5(1.8)$ & $<0.0001$ \\
\hline Birth weight $z$ score (mean) & 10,591 & $0.004(0.974)$ & $0.364(1.451)$ & $1.092(1.293)$ & $0.221(1.058)$ & $<0.0001$ \\
\hline Macrosomia $(n)$ & 10,591 & $1,171(12)$ & $8(20)$ & $19(36)$ & $66(18)$ & $<0.0001$ \\
\hline LGA $(n)$ & 10,591 & $962(9.5)$ & $10(25)$ & $22(42)$ & $62(17)$ & $<0.0001$ \\
\hline \multicolumn{7}{|l|}{ Offspring characteristics at age 9-11 years } \\
\hline $\mathrm{BMI} z$ score & 6,842 & $-0.006(0.991)$ & $0.129(1.280)$ & $0.302(1.225)$ & $0.137(1.041)$ & 0.032 \\
\hline General overweight/obesity ${ }^{\mathrm{b}}(n)$ & 6,842 & $1,481(23)$ & $6(23)$ & $12(30)$ & $71(31)$ & 0.035 \\
\hline Waist circumference $z$ score & 6,854 & $0.000(0.122)$ & $0.008(0.175)$ & $0.038(0.167)$ & $0.017(0.123)$ & 0.0013 \\
\hline Central overweight/obesity ${ }^{\mathrm{c}}(n)$ & 6,854 & $2,480(38)$ & $8(31)$ & $19(48)$ & $107(46)$ & 0.15 \\
\hline Fat mass $z$ score & 6,771 & $-0.006(0.989)$ & $0.202(1.370)$ & $0.099(0.922)$ & $0.172(1.002)$ & 0.036 \\
\hline Type 1 diabetes $(n)$ & 6,259 & $12(0.2)$ & 0 & 0 & $1(0.5)$ & 0.39 \\
\hline
\end{tabular}

The number of maternal-offspring pairs (pregnancies) varied from 6,771 to 10,591 for different characteristics

${ }^{a} p$ value for null hypothesis of no difference across the four groups (i.e. $3 d f$ )

${ }^{\mathrm{b}}$ Defined according to International Obesity Taskforce age- and sex-specific thresholds [17]

${ }^{\mathrm{c}}$ Defined as waist circumference $\geq 90$ th percentile using British childhood age- and sex-specific thresholds [19]

LGA, large for gestational age (>90th percentile of the birth weight $z$ score standardised for sex and gestational age)

Table 3 presents the multivariable associations of maternal diabetes/glycosuria status with offspring BMI, waist circumference and fat mass at age $9-11$ years. Similar patterns of association were seen for BMI and waist circumference, both gestational diabetes and glycosuria being positively associated with mean levels of these and with odds of general and central obesity in the basic model (model 1) and in the model additionally adjusting for maternal socioeconomic position, age, parity, smoking in pregnancy and mode of delivery (model 2). However, because of small numbers, the association with gestational diabetes was imprecise. Further adjustment for maternal prepregnancy BMI (model 3) resulted in attenuation, particularly of the associations with gestational diabetes.
Glycosuria was also positively associated with mean fat mass in all three models. Existing diabetes was not associated with BMI or waist circumference or with obesity. Adjustment for birthweight (either as a continuous variable or as macrosomia) and for number of urine tests completed during the mothers' pregnancy did not alter any of the associations in Table 3.

When analyses were repeated removing the nine motheroffspring pairs for whom mothers had existing diabetes but were not being treated with insulin, the results did not differ from any of those presented. Similarly, when analyses were repeated removing the 13 mother-offspring pairs in which the offspring had evidence of type 1 diabetes (treatment with insulin), the results did not differ from those presented. 
Table 2 Multivariable associations of maternal diabetes/glycosuria status with birth weight and macrosomia $(n=10,591)$

\begin{tabular}{|c|c|c|c|}
\hline \multirow[t]{2}{*}{ Outcome } & \multicolumn{3}{|c|}{ Mean difference/odds ratio $(95 \% \mathrm{CI})$} \\
\hline & Model $1^{\mathrm{a}}$ & Model $2^{b}$ & Model $3^{\mathrm{c}}$ \\
\hline \multicolumn{4}{|c|}{ Mean difference in sex- and gestational age-standardised birth weight $z$ score } \\
\hline Healthy $(n=10,126)$ & 0 & 0 & 0 \\
\hline Existing diabetes $(n=40)$ & $0.36(0.06-0.67)$ & $0.36(0.06-0.67)$ & $0.33(0.03-0.63)$ \\
\hline Gestational diabetes $(n=53)$ & $1.10(0.83-1.36)$ & $1.05(0.79-1.32)$ & $0.92(0.65-1.18)$ \\
\hline Glycosuria $(n=372)$ & $0.22(0.12-0.32)$ & $0.22(0.12-0.32)$ & $0.19(0.09-0.29)$ \\
\hline \multicolumn{4}{|l|}{ Odds ratio for macrosomia } \\
\hline Healthy $(n=10,126)$ & 1 & 1 & 1 \\
\hline Existing diabetes $(n=40)$ & $4.26(1.84-9.97)$ & $3.78(1.63-8.78)$ & $3.56(1.53-8.28)$ \\
\hline Gestational diabetes $(n=53)$ & $8.50(4.53-15.64)$ & $8.41(6.82-12.80)$ & $5.50(1.18-10.30)$ \\
\hline Glycosuria $(n=372)$ & $1.70(1.28-2.25)$ & $1.68(1.26-2.25)$ & $1.58(1.18-2.12)$ \\
\hline
\end{tabular}

${ }^{a}$ Model 1: Controlled for sex and gestational age — this control is achieved for birth weight $z$ score because these are standardised z-scores for sex and gestational age; for outcome of macrosomia control is achieved by multivariable adjustment for sex and gestational age in the multivariable model

${ }^{\mathrm{b}}$ Model 2: as model 1 and with additional adjustment for maternal age, social class, parity, smoking during pregnancy and mode of delivery

${ }^{\mathrm{c}}$ Model 3: as model 2 and with additional adjustment for maternal prepregnancy BMI

\section{Discussion}

In this general population sample of women who were recruited during their pregnancies in the early 1990s in the UK, we found all three of existing diabetes, gestational diabetes and glycosuria pregnancy to be positively associated with mean birthweight and macrosomia. Women with existing diabetes and gestational diabetes had 8.5- and 4.3fold increased risk of having an infant with macrosomia compared with women who had no evidence of diabetes or glycosuria during pregnancy. The increased risk associated with glycosuria was more modest at 1.7. Gestational diabetes and glycosuria were associated with greater offspring mean BMI and waist circumference, and with increased odds of general or central overweight/obesity, at age 9-11. However, the associations of gestational diabetes with these outcomes were imprecisely estimated because of small numbers, and attenuated to null with adjustment for maternal prepregnancy BMI. Existing diabetes was not associated with greater risk of overweight/obesity in offspring in later life in this study.

The main limitations of this study are the small numbers with existing or diagnosed gestational diabetes; our lack of knowledge about exactly how gestational diabetes was diagnosed; lack of knowledge about how women with diabetes (existing or gestational) were treated during the index pregnancy; and the probability that our category of women with glycosuria included a heterogeneous group of women, including those with undiagnosed gestational diabetes and hyperglycaemia, but also some with blood glucose levels in the normal range. Thus, the associations we have observed with glycosuria may be an underestimation of the true association of maternal hyperglycaemia with offspring adiposity because of non-differential measurement error. We are unable to examine the association of maternal diabetes/glycosuria with offspring fasting insulin or glucose currently in this study. However, these outcomes are now being assessed in the children who are currently in adolescence/young adulthood and will be available in the future.

The positive associations of gestational diabetes and glycosuria with later offspring obesity provide some support for the long-term effects of developmental overnutrition. Our results add to the limited literature in this area for non-Pima populations. In a study of 524 US women, all of whom were at high risk of gestational diabetes, exposure to mild diet-treated gestational diabetes ( $n=58$ cases) was not associated with mean BMI or obesity in offspring assessed up to age 8-10 years [27]. In the larger US Growing Up Today Study ( $n=14,881$ participants; 465 with gestational diabetes), results were similar to ours [28]. Maternal retrospective report of gestational diabetes (up to 40 years later) was associated with being overweight at age 9-14 years (odds ratio 1.4; 95\% CI 1.1-2.0) [28]. However, this attenuated towards the null upon adjustment for maternal BMI (odds ratio 1.2; 95\% CI 0.8-1.7). Adjustment for maternal BMI in that study, as well as our own, may represent overadjustment since this may be part of the developmental overnutrition mechanism [29]. The role of maternal obesity in fetal overnutrition is supported by results from a Danish study in which offspring of women with type 2 diabetes risk factors (obesity and family 
Table 3 Multivariable associations of maternal diabetes/glycosuria status with fat mass at age 9 - 11 years $(n=6,771-6,854)$

\begin{tabular}{|c|c|c|c|}
\hline \multirow[t]{2}{*}{ Outcome } & \multicolumn{3}{|c|}{ Mean difference or odds ratio in adiposity measurements at age 9 -11years $(95 \% \mathrm{CI})$} \\
\hline & Model $1^{\mathrm{a}}$ & Model $2^{\mathrm{b}}$ & Model $3^{\mathrm{c}}$ \\
\hline \multicolumn{4}{|c|}{ Mean difference in sex- and age-standardised BMI $z$ score } \\
\hline Healthy $(n=6,544)$ & 0 & 0 & 0 \\
\hline Existing diabetes $(n=26)$ & $0.15(-0.23,0.53)$ & $0.10(-0.27,0.50)$ & $0.05(-0.33,0.44)$ \\
\hline Gestational diabetes $(n=40)$ & $0.32(0.01,0.63)$ & $0.33(0.01,0.64)$ & $0.01(-0.30,0.63)$ \\
\hline Glycosuria $(n=232)$ & $0.14(0.01,0.27)$ & $0.14(0.02,0.29)$ & $0.09(-0.05,0.22)$ \\
\hline \multicolumn{4}{|l|}{ Odds ratio for overweight/obesity } \\
\hline Healthy $(n=6,544)$ & 1 & 1 & 1 \\
\hline Existing diabetes $(n=26)$ & $1.00(0.40,2.49)$ & $0.98(0.39,2.47)$ & $0.95(0.36,2.58)$ \\
\hline Gestational diabetes $(n=40)$ & $1.50(0.76,2.97)$ & $1.51(0.76,2.98)$ & $0.62(0.32,1.23)$ \\
\hline Glycosuria $(n=232)$ & $1.50(1.12,2.00)$ & $1.53(1.13,2.02)$ & $1.35(1.00,1.82)$ \\
\hline \multicolumn{4}{|c|}{ Outcome $=$ Mean difference in sex and age standardised waist circumference $z$ score } \\
\hline Healthy $(n=6,556)$ & 0 & 0 & 0 \\
\hline Existing diabetes $(n=26)$ & $0.01(-0.04,0.06)$ & $0.00(-0.05,0.05)$ & $0.00(-0.04,0.05)$ \\
\hline Gestational diabetes $(n=40)$ & $0.04(0.00,0.08)$ & $0.05(0.01,0.09)$ & $0.00(-0.04,0.06)$ \\
\hline Glycosuria (232) & $0.02(0.00,0.03)$ & $0.02(0.01,0.03)$ & $0.01(0.00,0.03)$ \\
\hline \multicolumn{4}{|c|}{ Outcome $=$ Odds ratio for central adiposity } \\
\hline Healthy $(n=6,556)$ & 1 & 1 & 1 \\
\hline Existing diabetes $(n=26)$ & $0.71(0.31,1.63)$ & $0.69(0.30,1.61)$ & $0.60(0.26,1.40)$ \\
\hline Gestational diabetes $(n=40)$ & $1.58(0.85,2.95)$ & $1.50(0.81,2.79)$ & $1.00(0.55,1.85)$ \\
\hline Glycosuria $(n=232)$ & $1.40(1.07,1.82)$ & $1.39(1.06,1.82)$ & $1.31(1.00,1.72)$ \\
\hline \multicolumn{4}{|c|}{ Mean difference in sex- and age-standardised fat mass $z$ score } \\
\hline Healthy $(n=6,478)$ & 0 & 0 & 0 \\
\hline Existing diabetes $(n=26)$ & $0.14(-0.21,0.48)$ & $0.08(-0.26,0.42)$ & $0.02(-0.31,0.35)$ \\
\hline Gestational diabetes $(n=38)$ & $0.07(-0.21,0.36)$ & $0.06(-0.22,0.34)$ & $-0.16(-0.43,0.11)$ \\
\hline Glycosuria $(n=229)$ & $0.18(0.06,0.30)$ & $0.17(0.06,0.29)$ & $0.12(0.01,0.23)$ \\
\hline
\end{tabular}

${ }^{\text {a }}$ Model 1: controlled for sex and age at outcome assessment — by standardisation of outcome — and adjustment gestational age. In addition, in models with fat mass as outcome there was adjustment for height and height squared at age 9-11 years

${ }^{\mathrm{b}}$ Model 2: as model 1 and with additional adjustment for maternal age, social class, parity, smoking during pregnancy and mode of delivery

${ }^{\mathrm{c}}$ Model 3: as model 2 and with additional adjustment for maternal prepregnancy BMI

history) but without gestational diabetes $(n=168)$ had mean BMI $\left(23.7 \mathrm{~kg} / \mathrm{m}^{2}\right)$ similar to that of women who were positive for gestational diabetes on their glucose tolerance test ( $n=141$; mean offspring BMI $23.8 \mathrm{~kg} / \mathrm{m}^{2}$ ) and those with existing type 1 diabetes $(n=160$; mean offspring BMI $23.5 \mathrm{~kg} / \mathrm{m}^{2}$ ), with all three being greater than the mean BMI of offspring whose mothers had no risk factors and no evidence of gestational, type 1 or type 2 diabetes in pregnancy $\left(n=120\right.$; mean BMI $22.4 \mathrm{~kg} / \mathrm{m}^{2}$ ) [30].

In the absence of universal screening for gestational diabetes with oral glucose tolerance tests in this study population, it is likely that some of women with glycosuria would fulfil current criteria for a diagnosis of gestational diabetes. This is supported by the low prevalence of gestational diabetes $(0.4 \%)$ in this study sample compared with prevalences of $1.2 \%$ and $1.8 \%$ among UK women who were screened, for research purposes, with fasting glucose and/or oral glucose tolerance tests, respectively, in the early 1990s [31, 32]. Women with glycosuria (who were not diagnosed with gestational diabetes) will not have been treated in any way to reduce their glycaemia and therefore fetal overnutrition may have occurred. Interestingly, the lack of attenuation to the null in this group with adjustment for maternal BMI suggests that higher maternal glucose levels, independent of adiposity, may be a risk factor for offspring adiposity.

In addition to fetal overnutrition as an explanation, it is also possible that a genetic or lifestyle predisposition to greater BMI and obesity in mothers results in their hyperglycaemia and hence glycosuria, and is inherited (genetic) or adopted (lifestyle) by their offspring, thus resulting in greater weight at birth (genes only) and throughout life. Studies of siblings among Pima Indians suggest that in that population the association of maternal gestational hyper- 
glycaemia with later offspring obesity is likely to be explained by specific intrauterine effects in addition to any genetic or behavioural mechanisms [10]. While some mothers in the ALSPAC study did have more than one live birth during the 2-year recruitment period, numbers of siblings within the study sample are too small to undertake within-sibling analyses.

Existing diabetes in this study was not associated with increased adiposity in offspring in later life. Given the mean age at diagnosis of diabetes in these women (12 years) and the fact that the majority were treated with insulin, we can assume that most of these women had type 1 diabetes. Studies to date that have examined the association of existing maternal type 1 diabetes with future offspring BMI or other markers of adiposity have produced varied results, with some showing greater BMI/adiposity in offspring of such mothers [30, 33] and others, like ours, showing no association [34, 35]. This variation could be due to small sample sizes in all of these studies, the number of mothers with existing diabetes in each study being 17 [35], 26 (our study), 61 [34], 75 [33] and 160 [30]. In addition to these studies, a recent study demonstrated that the adult offspring of mothers with existing type 1 diabetes during their pregnancy $(n=15)$ had similar mean BMI, waist/hip ratio and total fat mass to offspring whose fathers had type 1 diabetes $(n=15)$ [36].

A null or weaker association of existing diabetes with future offspring adiposity compared with that for gestational diabetes/hyperglycaemia may occur for a number of reasons. First, most studies to date, including ours, have had small numbers of mothers with existing diabetes, which makes the association difficult to interpret. Second, existing diabetes may be better controlled throughout the whole of pregnancy than newly occurring gestational diabetes/hyperglycaemia, and thus the developing fetus will be exposed to less extreme maternal glycaemia than fetuses of mothers with gestational diabetes/hyperglycaemia. In general, gestational diabetes is diagnosed from 24 weeks onwards and in our study many women with gestational diabetes may have been undiagnosed and therefore untreated. The small group of women in our study with existing diabetes may have been particularly well controlled. This would be consistent with the relatively modest increase in birthweight (SD 0.36) seen in our study for existing diabetes when gestational age was taken into account. The similarity in adiposity levels in offspring of mothers and fathers with type 1 diabetes provides some support for a genetic or shared familial environment mechanism, rather than an intrauterine mechanism linking these two [36].

Our study provides some evidence for a long-term effect of developmental overnutrition resulting from maternal hyperglycaemia during pregnancy on the risk of obesity in future offspring. If these findings are replicated in larger studies with more detailed measurements of gestational glycaemia they will further emphasise the importance of controlling maternal glycaemia in pregnancy as a means of preventing an intergenerational acceleration of the obesity and diabetes epidemic [11].

Acknowledgements We are extremely grateful to all the families who took part in this study, the midwives for their help in recruiting them, and the whole ALSPAC team, which includes interviewers, computer and laboratory technicians, clerical workers, research scientists, volunteers, managers, receptionists and nurses. The work presented in this paper was funded by a grant from United States National Institutes of Health (NIH): National Institute of Diabetes and Digestive and Kidney Diseases (R01 DK077659). The Medical Research Council (MRC), the Wellcome Trust and the University of Bristol provide core funding support for ALSPAC. The MRC and the University of Bristol provide core funding for the MRC Centre of Causal Analyses in Translational Epidemiology. A. Fraser is funded by a UK Medical Research Council research fellowship. The views expressed in this paper are those of the authors and not necessarily those of any funding body or others whose support is acknowledged. The funders had no role in study design, data collection and analysis, decision to publish, or preparation of the manuscript.

Duality of interest The authors declare that there is no duality of interest associated with this manuscript.

\section{References}

1. Jovanovic L, Pettitt DJ (2001) Gestational diabetes mellitus. JAMA 286:2516-2518

2. Catalano PM, Thomas A, Huston-Presley L, Amini SB (2003) Increased fetal adiposity: a very sensitive marker of abnormal in utero development. Am J Obstet Gynecol 189:1698-1704

3. Metzger BE, Lowe LP, Dyer AR et al (2008) Hyperglycemia and adverse pregnancy outcomes. N Engl J Med 358:1991-2002

4. Pedersen J (1954) Weight and length at birth of infants of diabetic mothers. Acta Endocrinol 16:330-342

5. Freinkel N (1980) Banting Lecture 1980. Of pregnancy and progeny. Diabetes 29:1023-1035

6. Dabelea D (2007) The predisposition to obesity and diabetes in offspring of diabetic mothers. Diabetes Care 30:S169-S174

7. Pettitt DJ, Baird HR, Aleck KA, Bennett PH, Knowler WC (1983) Excessive obesity in offspring of Pima Indian women with diabetes during pregnancy. N Engl J Med 308:242-245

8. Pettitt DJ, Knowler WC, Bennett PH, Aleck KA, Baird HR (1987) Obesity in offspring of diabetic Pima Indian women despite normal birth weight. Diabetes Care 10:76-80

9. Pettitt DJ, Nelson RG, Saad MF, Bennett PH, Knowler WC (1993) Diabetes and obesity in the offspring of Pima Indian women with diabetes during pregnancy. Diabetes Care 16:310-314

10. Dabelea D, Hanson RL, Lindsay RS et al (2000) Intrauterine exposure to diabetes conveys risks for type 2 diabetes and obesity: a study of discordant sibships. Diabetes 49:2208-2211

11. Ebbeling CB, Pawlak DB, Ludwig DS (2002) Childhood obesity: public-health crisis, common sense cure. Lancet 360:473-482

12. Hanna FW, Peters JR, Harlow J, Jones PW (2008) Gestational diabetes screening and glycaemic management: national survey on behalf of the Association of British Clinical Diabetologists. Q J Med 101:777-784

13. National Institute for Health and Clinical Excellence (2008) Antenatal care: routine care for the healthy pregnant woman (NICE Clinical Guideline CG62). RCOG Press, London 
14. American Diabetes Association (2004) Gestational diabetes mellitus: position statement. Diabetes Care 27:S88-S90

15. Scott DA, Loveman E, McIntyre L, Waugh N (2002) Screening for gestational diabetes: a systematic review and economic evaluation. Health Technol Assess 6:1-161

16. Gribble RK, Meier PR, Berg RL (1995) The value of urine screening for glucose at each prenatal visit. Obstet Gynecol $86: 405-410$

17. Buhling KJ, Elze L, Henrich W et al (2004) The usefulness of glycosuria and the influence of maternal blood pressure in screening for gestational diabetes. Eur J Obstet Gynecol Reprod Biol 113:145-148

18. Schytte T, Jorgensen LG, Brandslund I, Petersen PH, Andersen B (2004) The clinical impact of screening for gestational diabetes. Clin Chem Lab Med 42:1036-1042

19. Golding J, Pembrey M, Jones R (2001) ALSPAC-the Avon Longitudinal Study of Parents and Children. I. Study methodology. Paediatr Perinat Epidemiol 15:74-87

20. Lind T, Hytten FE (1972) The excretion of glucose during normal pregnancy. J Obstet Gynaecol Br Commonw 79:961-965

21. Lawlor DA, Timpson N, Harbord RM et al (2008) Exploring the developmental overnutrition hypothesis using parental-offspring associations and the FTO gene as an instrumental variable for maternal adiposity. The Avon Longitudinal Study of Parents and Children (ALSPAC). PloS Med 5:e33

22. Cole TJ, Bellizzi MC, Flegal KM, Dietz WH (2000) Establishing a standard definition for child overweight and obesity worldwide: international survey. BMJ 320:1240-1243

23. Zimmet P, Alberti G, Kaufman F et al (2007) The metabolic syndrome in children and adolescents. Lancet 369:2059-2061

24. McCarthy HD, Jarrett KV, Crawley HF (2001) The development of waist circumference percentiles in British children aged 5.0 16.9 y. Eur J Clin Nutr 55:902-907

25. Royston P (2004) Multiple imputation of missing values. Stata J 4:227-241

26. Rogers IS, Ness AR, Steer CD, Wells JC, Emmett PM, Reilly JR et al (2006) Associations of size at birth and dual-energy X-ray absorptiometry measures of lean and fat mass at 9 to $10 \mathrm{y}$ of age. Am J Clin Nutr 84:739-747

27. Whitaker RC, Pepe MS, Seidel KD, Wright JA, Knopp RH (1998) Gestational diabetes and the risk of offspring obesity. Pediatrics 101:e9

28. Gillman MW, Rifas-Shiman S, Berkey CS, Field AE, Colditz GA (2003) Maternal gestational diabetes, birth weight, and adolescent obesity. Pediatrics 111:e221-e226

29. Gillman MW (2004) A life course approach to obesity. In: Kuh D, Ben-Shlomo Y (eds) A life course approach to chronic disease epidemiology, 2nd edn. Oxford University Press, Oxford, pp 189217

30. Clausen TD, Mathiesen ER, Hansen $T$ et al (2008) High prevalence of type 2 diabetes and pre-diabetes in adult offspring of women with gestational diabetes mellitus or type 1 diabetes: the role of intrauterine hyperglycemia. Diabetes Care 31:340-346

31. Janghorbani M, Stenhouse E, Jones RB, Millward A (2006) Gestational diabetes mellitus in Plymouth, UK: prevalence, seasonal variation and associated factors. J Reprod Med 51:128-134

32. Koukkou E, Taub N, Jackson P, Metcalfe G, Cameron M, Lowy C (1995) Difference in prevalence of gestational diabetes and perinatal outcome in an innercity multiethnic London population. Eur J Obstet Gynecol Reprod Biol 59:153-157

33. Weiss PA, Scholz HS, Haas J, Tamussino KF, Seissler J, Borkenstein MH (2000) Long-term follow-up of infants of mothers with type 1 diabetes: evidence for hereditary and nonhereditary transmission of diabetes and precursors. Diabetes Care 23:905-911

34. Manderson JG, Mullan B, Patterson CC, Hadden DR, Traub AI, McCance DR (2002) Cardiovascular and metabolic abnormalities in the offspring of diabetic pregnancy. Diabetologia 45:991-996

35. Hunter WA, Cundy T, Rabone D et al (2004) Insulin sensitivity in the offspring of women with type 1 and type 2 diabetes. Diabetes Care 27:1148-1152

36. Sobngwi E, Boudou P, Mauvais-Jarvis F et al (2003) Effect of a diabetic environment in utero on predisposition to type 2 diabetes. Lancet 361:1861-1865 